TRANSACTIONS OF THE

AMERICAN MATHEMATICAL SOCIETY

Volume 359, Number 4, April 2007, Pages 1577-1592

S 0002-9947(06)03985-7

Article electronically published on October 16, 2006

\title{
A COMPLEX FROBENIUS THEOREM, MULTIPLIER IDEAL SHEAVES AND HERMITIAN-EINSTEIN METRICS ON STABLE BUNDLES
}

\author{
BEN WEINKOVE
}

\begin{abstract}
A complex Frobenius theorem is proved for subsheaves of a holomorphic vector bundle satisfying a finite generation condition and a differential inclusion relation. A notion of 'multiplier ideal sheaf' for a sequence of Hermitian metrics is defined. The complex Frobenius theorem is applied to the multiplier ideal sheaf of a sequence of metrics along Donaldson's heat flow to give a construction of the destabilizing subsheaf appearing in the DonaldsonUhlenbeck-Yau theorem, in the case of algebraic surfaces.
\end{abstract}

\section{INTRODUCTION}

In [Ko], J.J. Kohn introduced the notion of 'subelliptic multipliers' in his work on the $\bar{\partial}$-Neumann problem on pseudo-convex domains in order to prove subelliptic estimates on the boundary under certain conditions. He believed that his techniques would have wide applications in deriving estimates by algebraic methods. Following this line of approach, Nadel $\mathrm{Na}$ defined the multiplier ideal sheaf, and used it to prove the existence of Kähler-Einstein metrics on certain Fano manifolds with symmetries (his proofs were later simplified in [DeKo] ). Given a plurisubharmonic function $\phi$ on the manifold, the multiplier ideal sheaf of $\phi$ is the sheaf of germs of holomorphic functions $f$ satisfying

$$
\int|f|^{2} e^{-\phi}<\infty
$$

The sheaf measures the singularities of $\phi$. The concept of the multiplier ideal sheaf, which had already been considered implicitly in the work of Bomberi [Bo], Skoda [Sk and Siu [S1], is an important one in algebraic geometry, and has led to some important breakthroughs, such as the proof by Siu [S3] of the big Matsusaka theorem and effective results on very ampleness (e.g. [S4, De]).

In a number of lectures and seminars ([S5] for example), Y-T. Siu has advocated that generalized multiplier ideal sheaves have many applications to problems in partial differential equations and geometry. A multiplier ideal sheaf should measure the position and extent of the failure of an a priori estimate. A key concept is that the sheaf $\mathcal{I}$ should satisfy a differential inclusion relation which arises from known

Received by the editors January 18, 2005.

2000 Mathematics Subject Classification. Primary 53C07.

This work was carried out while the author was a Ph.D. student at Columbia University, supported by a graduate fellowship. 
estimates. In Kohn's case $(\underline{\mathrm{Ko}}$, Proposition 4.7.(G)) the differential relation takes the form

$$
\partial \mathcal{I} \subset \mathcal{M}
$$

where $\mathcal{M}$ is Kohn's 'multiplier module'. In this paper, we look at the notion of multiplier ideal sheaves in the context of the problem of the existence of HermitianEinstein metrics on stable bundles over compact Kähler manifolds. It is our hope that this may provide some clues as to how to proceed in other situations. The problem of existence of Hermitian-Einstein metrics has been solved over curves by Narasimhan and Seshadri [NS] (see also [D1] for an alternative proof), over algebraic surfaces by Donaldson D2 and over general compact Kähler manifolds by Uhlenbeck and Yau [UY]. In [UY], using a continuity method, it is shown that proving the existence of an Hermitian-Einstein metric can be reduced to proving a certain a priori estimate. If this estimate fails to hold, then they show that there exists a destabilizing subsheaf which contradicts the assumption that the original bundle was stable.

We give an alternative construction of the destabilizing subsheaf in the case of algebraic surfaces, following the ideas of Siu. Instead of the continuity method, we use the Donaldson heat flow (see section 3 ) which is roughly equivalent. The crucial estimate is the $C^{0}$ estimate for this flow of metrics. Abusing terminology, we define a 'multiplier ideal sheaf', picking out those directions in which the metric does not blow up. We show that, using a bound on the contracted curvature $\Lambda F$ along the flow, the multiplier ideal sheaf $\mathcal{F}$ satisfies the differential inclusion relation

$$
\bar{\partial} \mathcal{F} \subset \mathcal{F} \otimes T^{0,1} \text {. }
$$

The key property that the sheaf must satisfy is coherence. We show this using the differential inclusion relation and a complex Frobenius theorem which is given as follows.

Let $E$ be a holomorphic vector bundle of rank $r$ over a complex manifold $X$ of complex dimension $n$. Denote the 'partial connection' associated to the holomorphic structure on $E$ by $\bar{\partial}$. Denote by $\mathcal{O}, \mathcal{O}_{R}$ and $\mathcal{C}^{\infty}$ the sheaves of holomorphic, complex-valued real analytic and smooth functions on $X$, respectively. Let $\mathcal{E}, \mathcal{E}_{R}$ and $\mathcal{E}^{\infty}$ denote the sheaves of holomorphic, real analytic and $C^{\infty}$ sections of $E$ respectively. $T^{0,1}$ will denote the corresponding sheaf of $(0,1)$ forms on $X$.

Theorem 1. Let $\mathcal{F}_{R} \subseteq \mathcal{E}_{R}$ be a subsheaf satisfying

(i) $\mathcal{F}_{R}$ is locally finitely generated;

(ii) $\bar{\partial} \mathcal{F}_{R} \subseteq \mathcal{F}_{R} \otimes T^{0,1}$.

Then $\mathcal{F}_{R}$ is locally finitely generated by holomorphic sections of E. Denote by $\mathcal{F}$ the sheaf of $\mathcal{O}$-modules ker $\left.\bar{\partial}\right|_{\mathcal{F}_{R}}$. It follows that $\mathcal{F} \subset \mathcal{E}$ is coherent and

$$
\mathcal{F} \otimes \mathcal{O}_{R}=\mathcal{F}_{R}
$$

The corresponding result holds for a subsheaf $\mathcal{F}^{\infty} \subseteq \mathcal{E}^{\infty}$ if condition (i) is replaced by

(i)' For each $x$ in $X$, there exists an open set $U$ containing $x$ and a free resolution

$$
\cdots \longrightarrow\left(\mathcal{C}_{U}^{\infty}\right)^{d_{2}} \longrightarrow\left(\mathcal{C}_{U}^{\infty}\right)^{d_{1}} \longrightarrow\left(\mathcal{C}_{U}^{\infty}\right)^{d_{0}} \longrightarrow \mathcal{F}_{U}^{\infty} \longrightarrow 0
$$

for $d_{i} \geq 0$. 
If the subsheaf is locally free, this follows from the Koszul-Malgrange integrability theorem for vector bundles [KM], which is a linear version of the NewlanderNirenberg theorem for integrable almost complex structures [NN].

A result similar to the $C^{\infty}$ case of Theorem 1 has been proved by very different methods in a recent preprint of Pali $\mathrm{Pa}$. His theorem applies to sheaves of smooth functions with a $\bar{\partial}$ operator satisfying $\bar{\partial}^{2}=0$, and which admit local free resolutions of finite length. Pali does not assume that the sheaf is a subsheaf of a holomorphic vector bundle. In the case that it is, condition (i)' of Theorem 1 is slightly weaker than his condition, since we do not assume that the resolution is of finite length.

Note that the differential inclusion relation (ii) is not enough by itself to prove coherence. This can be seen by the following counterexample. Fix an open set $U$ in $X$ and consider the subsheaf with stalk $\mathcal{E}_{R, x}\left(\mathcal{E}_{x}^{\infty}\right)$ for $x$ in $U$ and the zero stalk otherwise. This satisfies condition (ii) but is clearly not coherent.

In our application of Theorem 1, we will only use the real analytic case. We define our multiplier ideal sheaf as follows. Suppose that $X$ is now a compact Kähler manifold, and let $\tilde{X}$ be an open subset of $X$. Let $\left\{H_{k}\right\}_{k=0}^{\infty}$ be a sequence of metrics on $E$ over $\tilde{X}$, and define endomorphisms $h_{k}=H_{0}^{-1} H_{k}$. Define a sheaf $\mathcal{F}_{R} \subseteq \mathcal{E}_{R}$ over $\tilde{X}$ by setting the sections over an open set $U \subset \tilde{X}$ to be

$$
\begin{gathered}
\mathcal{F}_{R}(U)=\left\{s \in \mathcal{E}_{R}(U) \mid \forall x \in U, \exists V \text { open with } x \in V \subset U\right. \text { and } \\
\left.\int_{V}|s|_{H_{k}}^{2} \rightarrow 0 \text { as } k \rightarrow \infty\right\},
\end{gathered}
$$

where integration is performed with respect to the volume form induced from the Kähler metric on $X$. We will call $\mathcal{F}_{R}$ the multiplier ideal sheaf associated to the sequence of metrics $\left\{H_{k}\right\}$. (For a different definition of a multiplier ideal sheaf for bundles, see $[\mathrm{DC}$.) If the sequence of metrics satisfies certain conditions, then we can prove the differential inclusion relation and apply Theorem 1 to obtain a coherent subsheaf of $E$. A bound is needed on the endomorphism $\Lambda F_{H_{k}}$. This is the contraction of the curvature of $H_{k}$ with the Kähler metric, and we denote it by $\hat{F}_{H_{k}}$.

Theorem 2. Suppose that there exists a positive constant $C$ and a real analytic endomorphism $h_{\infty}$ of $E$ such that the sequence of metrics $\left\{H_{k}\right\}_{k=0}^{\infty}$ satisfies

(i) $H_{k} \leq H_{0}$ for all $k$;

(ii) $i H_{k} \hat{F}_{H_{k}} \leq C H_{k}$ for all $k$;

(iii) $h_{k} \rightarrow h_{\infty}$ uniformly on compact subsets of $\tilde{X}$, for $h_{k}=H_{0}^{-1} H_{k}$.

Then the multiplier ideal sheaf $\mathcal{F}_{R}$ associated to $\left\{H_{k}\right\}$ defines a holomorphic coherent subsheaf $\mathcal{F} \subset \mathcal{E}$ over $\tilde{X}$.

Note that only the first two conditions in this theorem are needed to prove the differential inclusion relation. We will see how to use Theorem 2 to construct the destabilizing subsheaf in a proof of the theorem of Donaldson [D2].

Theorem 3. Let $X \subset \mathbf{C P}^{N}$ be a projective algebraic surface and let $\omega$ be the Kähler metric on $X$ induced from the Fubini-Study metric on $\mathbf{C P}^{N}$. Let $E$ be a holomorphic vector bundle of rank $r$ over $X$ which is stable with respect to the metric $\omega$. Then $E$ admits a Hermitian-Einstein metric.

It should be emphasized that the proof of Theorem 3 given here differs from existing proofs only in the construction of the destabilizing subsheaf and does not 
provide a simplification (in fact the given proof may be shortened; see [D2, [DoKr]). However, the method should help elucidate a certain point of view and provide a useful model for the further study of multiplier ideal sheaves. We use the YangMills heat flow and the associated flow of metrics, taking estimates from [D2, $\underline{\mathrm{DoKr}}$ and [UY]. The heat flow method has also been used by Simpson [Si] and de Bartolomeis and Tian DT to prove generalizations of the theorem of DonaldsonUhlenbeck-Yau.

Remark 1. The author has recently been made aware of a paper of Hong and Tian [HT] which describes the asymptotic behaviour and singularity set of the YangMills flow in higher dimensions. Using this result, it should be possible to extend the method of this paper to higher dimensions.

Remark 2. These results presented here do not completely realize the goals of Siu's program. The method is to work away from the finite set of points where the flow blows up, making use of Uhlenbeck's regularity results. One would hope to formulate a theory which can incorporate and come to grips directly with the singular set. Professor Siu has suggested that there should be a more general complex Frobenius theorem allowing for singularities.

The paper is arranged as follows. In section 2 we prove the complex Frobenius theorem. In section 3 we prove Theorem 2 by making use of the differential inclusion relation. In section 4 we give a proof of Theorem 3, using the Yang-Mills flow and Theorem 2.

Notation. We will use the notation from [D2]. In particular, the Kähler form $\omega$ on $X$ can be written in normal coordinates at a point as

$$
\omega=\frac{1}{2} i \sum_{j=1}^{2} d z^{j} \wedge d \bar{z}^{j},
$$

and the operator $\Lambda$ acts on $(1,1)$ forms at this point by

$$
\Lambda\left(\sum_{j, k=1}^{2} a_{j \bar{k}} d z^{j} \wedge d \bar{z}^{k}\right)=-2 i \sum_{j=1}^{2} a_{j \bar{j}} .
$$

If $\phi$ and $\psi$ are form-valued sections of $E$, define

$$
\langle\phi, \psi\rangle_{H}=\sum_{\alpha, \beta=1}^{r} H_{\alpha \bar{\beta}} \phi^{\alpha} \wedge \overline{\psi^{\beta}} .
$$

Note that if $H_{0}$ and $H$ are metrics on $E$, and $h=H_{0}^{-1} H$, then the associated curvatures $F_{H_{0}}$ and $F_{H}$ differ by

$$
F_{H}=F_{H_{0}}+\bar{\partial}\left(h^{-1} \partial_{0} h\right)
$$

For convenience, write $\hat{F}$ for $\Lambda F$.

\section{Complex Frobenius theorem}

In this section we give a proof of Theorem 1. We begin with the real analytic case. The statement is local, so it will suffice to prove the following. Let $\mathcal{F}_{R}$ be a subsheaf of $\mathcal{O}_{R}^{r}$ over the polydisc $U_{\eta}=\left\{z \in \mathbf{C}^{n}|| z_{j} \mid<\eta, j=1, \ldots, n\right\}$ for some $\eta>0$ satisfying the hypotheses (i) and (ii) of Theorem 1 . Let $f_{1}, \ldots, f_{q}$ be sections 
of $\mathcal{F}_{R}$ over $U_{\eta}$ generating the stalks $\mathcal{F}_{R, x}$ for all $x$ in $U_{\eta}$. We will show that there exists some $\eta^{\prime}$ with $0<\eta^{\prime}<\eta$ and sections $g_{1}, \ldots, g_{q}$ of $\mathcal{F}_{R}$ over $U_{\eta^{\prime}}$ such that $\bar{\partial} g_{i}=0$ for $i=1, \ldots, q$, with the $g_{i}$ generating $\mathcal{F}_{R, x}$ for all $x$ in $U_{\eta^{\prime}}$.

We will give a proof by induction on the number of variables $z_{1}, \ldots, z_{p}$ in which the generators are holomorphic.

Step 1. We begin by first showing that for small enough $\eta^{\prime}$ there exist sections $g_{1}, \ldots, g_{q}$ over $U_{\eta^{\prime}}$ satisfying $\partial_{\overline{1}} g_{i}=0$, and such that $g_{1}, \ldots, g_{q}$ generate $\mathcal{F}_{R, x}$ for all $x$ in $U_{\eta^{\prime}}$. By hypothesis, there exists a $q \times q$ matrix of real analytic functions $A_{1}$ on $U_{\eta}$ satisfying

$$
\left(\begin{array}{c}
\partial_{\overline{1}} f_{1} \\
\vdots \\
\partial_{\overline{1}} f_{q}
\end{array}\right)=A_{1}\left(\begin{array}{c}
f_{1} \\
\vdots \\
f_{q}
\end{array}\right)
$$

We will show that for small enough $\eta^{\prime}$ there exists a real analytic map $B: U_{\eta^{\prime}} \rightarrow$ $G L(q, \mathbf{C})$ satisfying

$$
\partial_{\overline{1}} B+B A=0 .
$$

To see that this will give the desired conclusion, set

$$
\left(\begin{array}{c}
g_{1} \\
\vdots \\
g_{q}
\end{array}\right)=B\left(\begin{array}{c}
f_{1} \\
\vdots \\
f_{q}
\end{array}\right)
$$

Since $B$ is invertible, the $g_{1}, \ldots, g_{q}$ generate $\mathcal{F}_{R, x}$ for $x$ in $U_{\eta^{\prime}}$ and differentiating the above gives

$$
\left(\begin{array}{c}
\partial_{\overline{1}} g_{1} \\
\vdots \\
\partial_{\overline{1}} g_{q}
\end{array}\right)=\left(\partial_{\overline{1}} B\right)\left(\begin{array}{c}
f_{1} \\
\vdots \\
f_{q}
\end{array}\right)+B A_{1}\left(\begin{array}{c}
f_{1} \\
\vdots \\
f_{q}
\end{array}\right)=0 .
$$

We will pull back the $f_{i}$ via the dilation map $\delta_{r}: \mathbf{C}^{n} \rightarrow \mathbf{C}^{n}$ given by $\left(z_{1}, z_{2}, \ldots, z_{n}\right)$ $\mapsto\left(r z_{1}, z_{2}, \ldots, z_{n}\right)$ for some $r<1$. Note that $A_{1}$ scales by the factor $r$. We will solve the problem for these new $f_{i}$ and then transform back.

Denote by $D_{\eta}$ the open disc of radius $\eta$ in $\mathbf{C}$. Multiply $A_{1}$ by a smooth cut-off function $\psi=\psi\left(\left|z_{1}\right|\right)$ with compact support in $D_{\eta / r}$ and equal to 1 on $D_{\eta / 2 r}$. We can then regard $A_{1}$ as a function on $\mathbf{C}$ with parameters $z_{2}, \bar{z}_{2}, \ldots, z_{n}, \bar{z}_{n}$ varying analytically in a small polycylinder which may be shrunk if necessary. Replacing these variables by $\zeta_{1}, \ldots, \zeta_{2 n-2}$, we can view $A_{1}$ as having holomorphic parameters $\zeta_{i}$. $A_{1}$ is smooth on $\mathbf{C}$, has compact support in $D_{\eta / r}$ and is real analytic in $D_{\eta / 2 r}$. By choosing $r$ small enough, we can suppose that, for a fixed $0<\epsilon<1$, the norm $\left\|A_{1}\right\|_{C^{\epsilon}}$ is as small as we like.

Now set $B=I+F$, so that (2.1) becomes

$$
\partial_{\overline{1}} F+(I+F) A_{1}=0 .
$$

We will use the following implicit function theorem to solve (2.2) for small real analytic $F$.

Theorem 2.1. Let $Y_{1}, Y_{2}$ and $Z$ be Banach spaces, and set $Y=Y_{1} \times Y_{2}$. Let $\Phi: Y \rightarrow Z$ be a smooth (holomorphic) map. Fix a point $\left(y_{1}, y_{2}\right)$ in $Y$. If the partial derivative $\left(D_{2} \Phi\right)\left(y_{1}, y_{2}\right): Y_{2} \rightarrow Z$ is surjective and admits a bounded right inverse 
$P: Z \rightarrow Y_{2}$, then there is a smooth (holomorphic) map $f$ from a neighbourhood of $y_{1}$ in $Y_{1}$ to a neighbourhood of $y_{2}$ in $Y_{2}$ such that

$$
\Phi(\eta, f(\eta))=\Phi\left(y_{1}, y_{2}\right) .
$$

It follows that if $\eta_{z}$ is a family of elements of $Y_{1}$ in the above neighbourhood of $y_{1}$, varying smoothly (holomorphically) in a parameter $z$, then $f\left(\eta_{z}\right)$ will also vary smoothly (holomorphically) in $z$.

Apply the theorem with $Y_{1}$ and $Z$ the Banach spaces of $q \times q$ matrix-valued functions on $\mathbf{C}$ with the norm \|\|$_{C^{\epsilon}}$ bounded, and $Y_{2}$ the same space with norm \|\|$_{C^{1+\epsilon}}$. Take $\left(y_{1}, y_{2}\right)=(0,0)$ and

$$
\Phi\left(A_{1}, F\right)=\partial_{\overline{1}} F+(I+F) A_{1} .
$$

Then $\left(D_{2} \Phi\right)\left(y_{1}, y_{2}\right): Y_{2} \rightarrow Z$ is given by

$$
\left(D_{2} \Phi\right)\left(y_{1}, y_{2}\right)(\alpha)=\partial_{\overline{1}} \alpha
$$

which has bounded right inverse $P: Z \rightarrow Y_{2}$ given by

$$
P(\beta)(z)=\frac{1}{2 \pi i} \int_{\mathbf{C}} \frac{\beta(w)}{w-z} d w \wedge d \bar{w} .
$$

Hence, if $\left\|A_{1}\right\|_{C^{\epsilon}}$ is small enough, then there exists $F=F\left(A_{1}\right)$ with $\|F\|_{C^{1+\epsilon}}$ small satisfying (2.2). Now since $A_{1}$ depends holomorphically on the parameters $\zeta_{i}$, and since $\Phi$ is holomorphic, the solution $F$ will also be holomorphic in these parameters. By making the norm of $A_{1}$ smaller if necessary, the solution $B=I+F$ will be invertible. Moreover, since $F$ is a solution of the elliptic equation

$$
\left(\partial_{\bar{z}_{1}}+\partial_{\bar{\zeta}_{1}}+\ldots+\partial_{\bar{\zeta}_{2 n-2}}\right) F+(I+F) A_{1}=0
$$

it follows that $F$ must also be analytic in the variables $z_{1}$ and $\bar{z}_{1}$ by the theorem that sufficiently smooth solutions to elliptic equations with real analytic coefficients are themselves real analytic. Replacing the $\zeta_{1}, \ldots, \zeta_{2 n-2}$ with $z_{2}, \ldots, \bar{z}_{n}$, we see that $F$ is analytic in $z_{1}, \bar{z}_{1}, \ldots, z_{n}, \bar{z}_{n}$. We have now completed the first step in the induction.

Step 2. Fix $p$ with $1 \leq p \leq n-1$. Our inductive hypothesis is that for $\eta^{\prime}$ small enough, there exist sections $f_{1}, \ldots, f_{q}$ over $U_{\eta^{\prime}}$ satisfying $\partial_{\bar{j}} f_{i}=0$ for $j=1, \ldots, p$ and generating $\mathcal{F}_{R, x}$ for $x$ in $U_{\eta^{\prime}}$. We will show that, for some $\eta^{\prime \prime}>0$, there exist sections $g_{1}, \ldots, g_{q}$ over $U_{\eta^{\prime \prime}}$ satisfying $\partial_{\bar{j}} g_{i}=0$ for $j=1, \ldots, p+1$ and such that $g_{1}, \ldots, g_{q}$ generate $\mathcal{F}_{R, x}$ for $x$ in $U_{\eta^{\prime \prime}}$.

We know that there exist real analytic functions of $q \times q$ matrices $A_{k}$ on $U_{\eta^{\prime}}$ for $k=1, \ldots, n$ satisfying

$$
\left(\begin{array}{c}
\partial_{\bar{k}} f_{1} \\
\vdots \\
\partial_{\bar{k}} f_{q}
\end{array}\right)=A_{k}\left(\begin{array}{c}
f_{1} \\
\vdots \\
f_{q}
\end{array}\right) .
$$

The inductive hypothesis implies that $A_{k}=0$ for $k=1, \ldots, p$. We will show that, for $\eta^{\prime \prime}$ small enough, there exists a real analytic function $B: U_{\eta^{\prime \prime}} \rightarrow G L(q, \mathbf{C})$, holomorphic in the variables $z_{1}, \ldots, z_{p}$, satisfying

$$
\partial_{\overline{p+1}} B+B A_{p+1}=0 .
$$


Then, as before, the sections $g_{i}$ given by

$$
\left(\begin{array}{c}
g_{1} \\
\vdots \\
g_{q}
\end{array}\right)=B\left(\begin{array}{c}
f_{1} \\
\vdots \\
f_{q}
\end{array}\right)
$$

will satisfy the required properties.

Before using the implicit function theorem, we must ensure that $A_{p+1}$ is holomorphic in $z_{1}, \ldots, z_{p}$. Note that in (2.3) for $k=p+1, A_{p+1}$ is an analytic function of all of the variables $z_{1}, \bar{z}_{1}, \ldots, z_{n}, \bar{z}_{n}$, whereas the $\partial_{\overline{p+1}} f_{i}$ and the $f_{i}$ do not depend on $\bar{z}_{1}, \ldots, \bar{z}_{p}$. Setting $\bar{z}_{1}=\ldots=\bar{z}_{p}=0$ we get an $A_{p+1}$ which satisfies (2.3) and is holomorphic in $z_{1}, \ldots, z_{p}$.

We can now finish the proof. Let $B=I+F$. We use the same method as before to solve

$$
\partial_{\overline{p+1}} F+(I+F) A_{p+1}=0
$$

for $F$ small, real analytic, and holomorphic in $z_{1}, \ldots, z_{p}$, given that $A_{p+1}$ is holomorphic in $z_{1}, \ldots, z_{p}$. After dilating the $z_{p+1}$ coordinate and multiplying by a cut-off function $\psi=\psi\left(\left|z_{p+1}\right|\right)$, we can take $A_{p+1}$ to be a smooth function on $\mathbf{C}$ in the $z_{p+1}$ variable, with the $z_{i}(i \neq p+1)$ regarded as parameters which vary in some small polycylinder. As before we can assume that $\left\|A_{p+1}\right\|_{C^{\epsilon}}$ is as small as we like. Apply the implicit function theorem to get a solution $F$ with $\|F\|_{C^{1+\epsilon}}$ small and which is analytic in $z_{p+1}, \bar{z}_{p+1}, \ldots, z_{n}, \bar{z}_{n}$ and holomorphic in the variables $z_{1}, \ldots, z_{p}$. This completes the induction, and the theorem for the real analytic case follows.

For the smooth case, we cannot apply the argument used in Step 2 to get an $A_{p+1}$ holomorphic in $z_{1}, \ldots, z_{p}$. We will replace this with another induction argument. Define a proposition $P_{k}$ for $1 \leq k \leq p$ as follows.

Proposition $\boldsymbol{P}_{\boldsymbol{k}}$. Let $\mathcal{G}^{\infty} \subset\left(\mathcal{C}^{\infty}\right)^{d}$ be a subsheaf over $U_{\delta}$ for some $\delta>0$, satisfying condition (i)' of the theorem. Suppose that $\mathcal{G}^{\infty}$ is generated over $U_{\delta}$ by sections $r_{1}, \ldots, r_{l}$, which are holomorphic in $z_{1}, \ldots, z_{k}$. Let $a_{1}, \ldots, a_{l}$ be smooth functions on $U_{\delta}$ satisfying

$$
\left(\partial_{\bar{j}} a_{1} \cdots \partial_{\bar{j}} a_{l}\right)\left(\begin{array}{c}
r_{1} \\
\vdots \\
r_{l}
\end{array}\right)=0, \quad \text { for } j=1, \ldots, k .
$$

Then there exists $\delta^{\prime}$ with $0<\delta^{\prime}<\delta$ and $\left(s_{1} \cdots s_{l}\right)$ in the $\mathcal{C}^{\infty}$ sheaf of relations $\mathcal{R}\left(r_{1}, \ldots, r_{l}\right)$ over $U_{\delta^{\prime}}$ with

$$
\partial_{\bar{j}}\left(a_{1}-s_{1}\right)=\ldots=\partial_{\bar{j}}\left(a_{l}-s_{l}\right)=0, \quad \text { for } j=1, \ldots, k .
$$

We will show that $P_{k}$ holds for $k=p$. Then since

$$
\partial_{\bar{j}} A_{p+1}\left(\begin{array}{c}
f_{1} \\
\vdots \\
f_{q}
\end{array}\right)=0, \quad \text { for } j=1, \ldots, p,
$$

we can apply this result to each row of $A_{p+1}$. Hence, after making permissible changes to $A_{p+1}$ and passing to a smaller open set, we can assume that $A_{p+1}$ is holomorphic in $z_{1}, \ldots, z_{p}$. We will need to use the main inductive hypothesis in the proof of this induction. 
Proof of $P_{1}$. By assumption, the sheaf of relations $\mathcal{R}\left(r_{1}, \ldots, r_{l}\right)$ is locally finitely generated. Suppose the generators are

$$
h_{1}=\left(h_{11} h_{12} \cdots h_{1 l}\right), \ldots, h_{m}=\left(h_{m 1} h_{m 2} \cdots h_{m l}\right) .
$$

Since $r_{1}, \ldots, r_{l}$ are holomorphic in $z_{1}$, and $\mathcal{R}\left(r_{1}, \ldots, r_{l}\right)$ satisfies (i)', we can apply the main inductive hypothesis in the case $p=1$ to this subsheaf. Hence we can assume that, after choosing $\delta^{\prime}$ small enough, the generators $h_{1}, \ldots, h_{m}$ are holomorphic in the variable $z_{1}$. Now there exist smooth functions $t_{1}, \ldots, t_{m}$ on $U_{\delta^{\prime}}$ satisfying

$$
\left(\partial_{\overline{1}} a_{1} \cdots \partial_{\overline{1}} a_{l}\right)=t_{1} h_{1}+\ldots+t_{m} h_{m} .
$$

Let $\hat{t}_{1}, \ldots, \hat{t}_{m}$ be smooth functions satisfying $\partial_{\overline{1}} \hat{t}_{i}=t_{i}$ (shrinking $\delta^{\prime}$ slightly). Define

$$
\left(s_{1} \cdots s_{l}\right)=\hat{t}_{1} h_{1}+\ldots+\hat{t}_{m} h_{m} .
$$

Then we see that $\left(s_{1} \cdots s_{l}\right) \in \mathcal{R}\left(r_{1}, \ldots, r_{l}\right)$ and

$$
\partial_{\overline{1}}\left(a_{1}-s_{1}\right)=\ldots=\partial_{\overline{1}}\left(a_{l}-s_{l}\right)=0,
$$

completing the proof of $P_{1}$.

Proof of $P_{k} \Rightarrow P_{k+1}$. We assume $1 \leq k \leq p-1$. Suppose that

$$
\left(\partial_{\bar{j}} a_{1} \cdots \partial_{\bar{j}} a_{l}\right)\left(\begin{array}{c}
r_{1} \\
\vdots \\
r_{l}
\end{array}\right)=0, \quad \text { for } j=1, \ldots, k+1 .
$$

By the inductive hypothesis, we can suppose, after making some allowed changes and taking $\delta^{\prime}$ small enough, that $\partial_{\bar{j}} a_{i}=0$ for $j=1, \ldots, k$ and $i=1, \ldots, l$. As before let the generators of $\mathcal{R}\left(r_{1}, \ldots, r_{l}\right)$ be

$$
h_{1}=\left(h_{11} h_{12} \cdots h_{1 l}\right), \ldots, h_{m}=\left(h_{m 1} h_{m 2} \cdots h_{m l}\right) .
$$

Since $r_{1}, \ldots, r_{l}$ are holomorphic in $z_{1}, \ldots, z_{k}$, by applying the main inductive hypothesis, we can assume that $h_{1}, \ldots, h_{m}$ are holomorphic in $z_{1}, \ldots, z_{k}$. There exist $t_{1}, \ldots, t_{m}$ smooth functions with

$$
\left(\partial_{\overline{k+1}} a_{1} \cdots \partial_{\overline{k+1}} a_{l}\right)=t_{1} h_{1}+\ldots+t_{m} h_{m} .
$$

Note that by applying $\partial_{\bar{j}}$ we get that

$$
\left(\partial_{\bar{j}} t_{1} \cdots \partial_{\bar{j}} t_{m}\right)\left(\begin{array}{c}
h_{1} \\
\vdots \\
h_{m}
\end{array}\right)=0, \quad \text { for } j=1, \ldots, k .
$$

We can apply the current inductive hypothesis to see that, after shrinking $\delta^{\prime}$, there exists $\left(s_{1}^{\prime} \cdots s_{m}^{\prime}\right)$ in $\mathcal{R}\left(h_{1}, \ldots, h_{m}\right)$ such that

$$
\partial_{\bar{j}}\left(t_{1}-s_{1}^{\prime}\right)=\ldots=\partial_{\bar{j}}\left(t_{m}-s_{m}^{\prime}\right)=0, \quad \text { for } j=1, \ldots, k .
$$

By replacing the $t_{i}$ by $t_{i}-s_{i}^{\prime}$, we can then assume that $\partial_{\bar{j}} t_{i}=0$ for $j=1, \ldots, k$. Let $\hat{t}_{i}$ solve $\partial_{\overline{k+1}} \hat{t}_{i}=t_{i}$ for $i=1, \ldots, m$, where the $\hat{t}_{i}$ are smooth and holomorphic in $z_{1}, \ldots, z_{k}$. Then, as before, set

$$
\left(s_{1} \cdots s_{l}\right)=\hat{t}_{1} h_{1}+\ldots+\hat{t}_{m} h_{m} .
$$


Then $\left(s_{1} \cdots s_{l}\right) \in \mathcal{R}\left(r_{1}, \ldots, r_{l}\right)$ and

$$
\partial_{\bar{j}}\left(a_{1}-s_{1}\right)=\ldots=\partial_{\bar{j}}\left(a_{l}-s_{l}\right)=0, \quad \text { for } j=1, \ldots, k+1 .
$$

This proves $P_{k+1}$.

It is now straightforward to complete the proof in the smooth case. To see that $\mathcal{F}=\left.\operatorname{ker} \bar{\partial}\right|_{\mathcal{F} \infty}$ is coherent and to establish

$$
\mathcal{F} \otimes \mathcal{C}^{\infty}=\mathcal{F}^{\infty}
$$

one can use the result of Malgrange [Ma on the flatness of $\mathcal{C}^{\infty}$ over $\mathcal{O}$, or apply Corollary 6.3.6 of [Ho].

\section{The MUlTiplier IDEAL SHEAF}

In this section, we give a proof of Theorem 2. Let $H_{k}$ be a sequence of metrics as given in the hypothesis of the theorem, defining the multiplier ideal sheaf $\mathcal{F}_{R}$ over $\tilde{X}$. We now prove the differential inclusion relation, using only conditions (i) and (ii) of the theorem.

Proposition 3.1. $\bar{\partial} \mathcal{F}_{R} \subseteq \mathcal{F}_{R} \otimes T^{0,1}$.

Proof. Let $x$ be in $\tilde{X}$, and let $U$ be an open set in $\tilde{X}$ containing $x$. Choose $V$ compact and $W$ open with $x \in W \subset V \subset U$ and such that there exists a smooth cut-off function $\psi$ with bounded derivatives supported in $V$ and equal to 1 in $W$. Suppose that $s$ satisfies

$$
\int_{U}|s|_{H_{k}}^{2} \rightarrow 0 \quad \text { as } k \rightarrow \infty .
$$

Then

$$
\begin{aligned}
\int_{W}|\bar{\partial} s|_{H_{k}}^{2}= & -i \int_{W} \Lambda\langle\bar{\partial} s, \bar{\partial} s\rangle_{H_{k}} \\
\leq & -i \int_{X} \Lambda\left\langle\psi h_{k} \bar{\partial} s, \bar{\partial} s\right\rangle_{H_{0}} \\
= & -i \int_{X} \Lambda\left\langle\psi\left(\partial_{0} h_{k}\right) \bar{\partial} s, s\right\rangle_{H_{0}}-i \int_{X} \Lambda\left\langle\psi h_{k} \partial_{0} \bar{\partial} s, s\right\rangle_{H_{0}} \\
& -i \int_{X} \Lambda\left\langle(\partial \psi) h_{k} \bar{\partial} s, s\right\rangle_{H_{0}} \\
= & -i \int_{X} \Lambda\left\langle\psi\left(h_{k}\right)^{-\frac{1}{2}}\left(\partial_{0} h_{k}\right) \bar{\partial} s,\left(h_{k}\right)^{\frac{1}{2}} s\right\rangle_{H_{0}} \\
& -i \int_{X} \Lambda\left\langle\psi\left(h_{k}\right)^{\frac{1}{2}} \partial_{0} \bar{\partial} s,\left(h_{k}\right)^{\frac{1}{2}} s\right\rangle_{H_{0}} \\
& -i \int_{X} \Lambda\left\langle(\partial \psi)\left(h_{k}\right)^{\frac{1}{2}} \bar{\partial} s,\left(h_{k}\right)^{\frac{1}{2}} s\right\rangle_{H_{0}} \\
\leq & C\left(\int_{V}\left|\left(h_{k}\right)^{-\frac{1}{2}} \partial_{0} h_{k}\right|_{H_{0}}^{2}\right)^{\frac{1}{2}}\left(\int_{U}|s|_{H_{k}}^{2}\right)^{\frac{1}{2}} \\
& +\left(\int_{V}|\partial \bar{\partial} s|_{H_{k}}^{2}\right)^{\frac{1}{2}}\left(\int_{U}|s|_{H_{k}}^{2}\right)^{\frac{1}{2}} \\
& +C\left(\int_{V}|\bar{\partial} s|_{H_{k}}^{2}\right)^{\frac{1}{2}}\left(\int_{U}|s|_{H_{k}}^{2}\right)^{\frac{1}{2}}
\end{aligned}
$$


where $C$ denotes a constant that does not depend on $k$. The last two terms in the last line clearly tend to zero as $k$ tends to infinity. To see that the first term in the last line also tends to zero, observe that

$$
\begin{aligned}
\int_{X}\left|\left(h_{k}\right)^{-\frac{1}{2}} \partial_{0} h_{k}\right|_{H_{0}}^{2} & =i \int_{X} \Lambda\left\langle\left(h_{k}\right)^{-1} \partial_{0} h_{k}, \partial_{0} h_{k}\right\rangle_{H_{0}} \\
& =i \int_{X} \Lambda\left\langle\bar{\partial}\left(\left(h_{k}\right)^{-1} \partial_{0} h_{k}\right), h_{k}\right\rangle_{H_{0}} \\
& =\int_{X}\left\langle\left(i \hat{F}_{H_{k}}-i \hat{F}_{H_{0}}\right), h_{k}\right\rangle_{H_{0}} \\
& \leq C
\end{aligned}
$$

where we have used the fact that the contracted curvature $i \hat{F}_{H_{k}}$ is bounded from above. This completes the proof.

In order to apply Theorem 1 we must use the hypothesis (iii) to show that $\mathcal{F}_{R}$ is locally finitely generated. This follows from the next lemma, which gives an alternative description of the multiplier ideal sheaf.

Lemma 3.2. Define a sheaf $\mathcal{S}_{\mathcal{R}}$ on $\tilde{X}$ by

$$
\mathcal{S}_{\mathcal{R}}(U)=\left\{s \in \mathcal{E}_{R}(U) \mid h_{\infty} s=0\right\} .
$$

Then $\mathcal{F}_{R}=\mathcal{S}_{\mathcal{R}}$.

Proof. Let $x$ be in $\tilde{X}$, and let $x \in U \subset \tilde{X}$. Choose $V$ compact and $W$ open such that $x \in W \subset V \subset U$. If $s$ is in $\mathcal{S}_{\mathcal{R}}(U)$, then

$$
\int_{W}|s|_{H_{k}}^{2}=\int_{W}\left\langle\left(h_{k}-h_{\infty}\right) s, s\right\rangle_{H_{0}} \rightarrow 0,
$$

as $k$ tends to infinity. This proves $\mathcal{S}_{\mathcal{R}} \subset \mathcal{F}_{R}$. On the other hand, suppose that

$$
\int_{U}|s|_{H_{k}}^{2} \rightarrow 0, \quad \text { as } k \rightarrow \infty \text {. }
$$

Then for any smooth section $t$ of $E$ over $U$,

$$
\begin{aligned}
\int_{V}\left\langle h_{\infty} s, t\right\rangle_{H_{0}} & =\int_{V}\left\langle\left(h_{\infty}-h_{k}\right) s, t\right\rangle_{H_{0}}+\int_{V}\langle s, t\rangle_{H_{k}} \\
& \leq \int_{V}\left\langle\left(h_{\infty}-h_{k}\right) s, t\right\rangle_{H_{0}}+\left(\int_{U}|s|_{H_{k}}^{2}\right)^{\frac{1}{2}}\left(\int_{V}|t|_{H_{k}}^{2}\right)^{\frac{1}{2}} \\
& \rightarrow 0
\end{aligned}
$$

as $k$ tends to infinity. Hence $h_{\infty} s=0$ on $W$, and the lemma is proved.

Since $h_{\infty}$ is real analytic, $\mathcal{F}_{R}$ is locally the kernel of a homomorphism between coherent sheaves of $\mathcal{O}_{R}$ modules, and so $\mathcal{F}_{R}$ is locally finitely generated. Applying Theorem 1 completes the proof.

\section{Hermitian Einstein metrics on stable Bundles}

For this section, we will assume the hypotheses of Theorem 3. The background material for this section can be found in D2] and DoKr] (see also S2 for a good exposition).

Let $H_{0}$ be a real analytic Hermitian metric on the fibres of $E$. We can obtain such a metric in the following way. Tensor $E$ with the hyperplane line bundle 
$L$ raised to a high power $K$ so that the holomorphic sections of $E \otimes L^{K}$ embed $X$ into a Grassmannian $G(r, M)$ of $r$-planes in $\mathbf{C}^{M}$, where $M$ is the dimension of $H^{0}\left(X, E \otimes L^{K}\right)$. Then, via this embedding, pull back the canonical metric on the universal bundle $U(r)$ over the Grassmannian to get a real analytic metric on $E \otimes L^{K}$. Then use the canonical real analytic metric on $L$ to get a real analytic metric $H_{0}$ on $E$.

Let $\lambda$ be the average of $\left(\operatorname{Tr} \hat{F}_{0}\right) / r$. We consider the flow of endomorphisms $h_{t}$ with $h_{0}=I d_{E}$ given by the flow

$$
\frac{\partial h_{t}}{\partial t}=-2 i h_{t}\left(\hat{F}_{t}-\lambda I\right)
$$

introduced by Donaldson D2] $\left(F_{t}\right.$ is the curvature of the metric $\left.H_{t}=H_{0} h_{t}\right)$. We call this the Donaldson heat flow. It is shown in D2 that solutions exist for all time. The flow is, roughly speaking, the same as the Yang-Mills flow up to gauge. In particular, if $A_{0}$ is the unitary connection associated to $H_{0}$ and the holomorphic structure on $E$, and if $g_{t}$ in the complexified gauge group $\mathcal{G}^{C}$ satisfies $g_{t}^{*} g_{t}=h_{t}$ (where $g_{t}^{*}$ denotes the adjoint of $g_{t}$ with respect to $\left.H_{0}\right)$, then $A_{t}=g_{t}\left(A_{0}\right)$ satisfies the gauge equivalent Yang-Mills flow

$$
\frac{\partial A_{t}}{\partial t}=-d_{A_{t}}^{*} F_{A_{t}}+d_{A_{t}}(\alpha(t))
$$

for $\alpha(t) \in \Omega^{0}\left(\mathbf{g}_{E}\right)$.

Make a (real analytic) conformal change to $H_{0}$ so that det $h_{t}=1$ along the flow. Also, normalize the metric on $X$ so that $\operatorname{Vol}(X)=2 \pi$. In the following, $L_{k}^{p}$ will denote the Sobolev space obtained by compactifying the space of smooth sections of the appropriate bundle with respect to the norm

$$
\|v\|_{L_{k}^{p}}=\sum_{i=0}^{k}\left\|\nabla^{i} v\right\|_{L^{p}}
$$

where the covariant derivative and $L^{p}$ norm are defined with respect to the Hermitian metric $H_{0}$ and the Kähler metric on $X$.

Suppose first that $\sup _{X}\left|h_{t}\right|_{H_{0}}$ is bounded uniformly in $t$. By the argument in D2, we have a $C^{1}$ bound and $L_{2}^{p}$ bounds for all $p$ for $h_{t}$. Hence, for a sequence of times $t_{k} \rightarrow \infty, H_{k}=H_{t_{k}}$ converges strongly in $L_{1}^{p}$ to a Hermitian metric $H_{\infty}$, which is itself in $L_{2}^{p}$. Then

$$
\hat{F}_{H_{k}}-\lambda I \rightarrow \hat{F}_{H_{\infty}}-\lambda I
$$

weakly in $L^{p}$. Now, Donaldson's functional (see [D2]) is bounded below when $E$ is stable (in fact, semi-stable), and calculating the functional along the flow gives

$$
\hat{F}_{H_{k}}-\lambda I \rightarrow 0
$$

strongly in $L^{2}$. Hence we get that $\hat{F}_{\infty}=\lambda I$, and, by elliptic estimates, $H_{\infty}$ is a smooth (and in fact real analytic) Hermitian-Einstein metric.

Suppose then that the $C^{0}$ norm of $h_{t}$ is unbounded along the flow. We will show that this leads to a contradiction. Take a sequence $h_{t_{k}}$ with the $C^{0}$ norm tending to infinity as $k$ tends to infinity. Then define normalized endomorphisms $h_{k}^{\prime}$ by

$$
h_{k}^{\prime}=\frac{h_{t_{k}}}{\sup _{X}\left|h_{t_{k}}\right|_{H_{0}}},
$$


and let $H_{k}^{\prime}=H_{0} h_{k}^{\prime}$. Then we have the following theorem, the proof of which is, for the most part, contained in [D2] and [DoKr].

Theorem 4.1. There exists a finite set of points $\left\{x_{1}, \ldots, x_{p}\right\} \subset X$ such that, after passing to a subsequence, $h_{k}^{\prime}$ converges uniformly on compact sets in $X \backslash\left\{x_{1}, \ldots, x_{p}\right\}$ to a real analytic endomorphism $h_{\infty}$.

Proof. Define $A_{k}=h_{k}^{\prime 1 / 2}\left(A_{0}\right)$. Then we have

(i) $F_{A_{k}}$ are bounded in $L^{2}$;

(ii) $\hat{F}_{A_{k}}$ are uniformly bounded;

(iii) $\nabla_{A_{k}} \hat{F}_{A_{k}} \rightarrow 0$ in $L^{2}$.

(i) follows from the fact that the Yang-Mills flow is the gradient flow for the Yang-Mills functional, (ii) follows from a maximum principle argument (see [D2]) and a proof of (iii) is given in $\mathrm{DoKr}$ (Proposition 6.2.14).

It is shown in DoKr] that there exists a set of points $\left\{x_{1}, \ldots, x_{p}\right\}$ in $X$ and a cover of $X \backslash\left\{x_{1}, \ldots, x_{p}\right\}$ by a system of balls such that, after passing to a subsequence, each connection $A_{k}$ has curvature with $L^{2}$ norm less than a small $\epsilon>0$. If this $\epsilon$ is small enough, then by a theorem of Uhlenbeck's [Uh, we can put the connections in Coulomb gauge over these balls. By elliptic estimates and a standard patching argument (see [DoKr] for details), there exist gauge transformations $v_{k}$ over the punctured manifold such that $v_{k} h_{k}^{\prime 1 / 2}\left(A_{0}\right)$ are bounded in $L_{2}^{2}$. Put $g_{k}=v_{k} h_{k}^{\prime 1 / 2}$, and let $\tilde{A}_{k}=g_{k}\left(A_{0}\right)$. Then if $\tilde{A}_{k}^{1,0}$ and $\tilde{A}_{k}^{0,1}$ are the $(1,0)$ and $(0,1)$ components of $\tilde{A}_{k}$ we have

$$
\begin{aligned}
& \tilde{A}_{k}^{1,0}=A_{0}^{1,0}+g_{k}^{-1}\left(\partial_{A_{0}} g_{k}\right), \\
& \tilde{A}_{k}^{0,1}=A_{0}^{0,1}+\left(\bar{\partial} g_{k}\right) g_{k}^{-1} .
\end{aligned}
$$

Since $g_{k}^{*} g_{k}=h_{k}^{\prime}$ we have that the $g_{k}$ are bounded uniformly, and from the above, they are bounded in $L_{3}^{2}$. Hence, after passing to a subsequence, the $g_{k}$ converge uniformly on compact subsets to $g_{\infty}$, from which it follows that $h_{k}^{\prime}$ converges uniformly on compact subsets to $h_{\infty}$. It remains to see that $h_{\infty}$ is real analytic. Now $\tilde{A}_{k}$ converges over the punctured manifold to $A_{\infty}$, weakly in $L_{2}^{2}$, and by condition (iii) we must have

$$
\nabla_{A_{\infty}} \hat{F}_{A_{\infty}}=0
$$

which is elliptic in a Coulomb gauge. The elliptic estimates give that $A_{\infty}$ is smooth. Since the coefficients of the equation are real analytic (using the fact that $\omega$ is real analytic), it follows that $A_{\infty}$ is real analytic. Now

$$
\begin{aligned}
& A_{\infty}^{1,0}=A_{0}^{1,0}+g_{\infty}^{-1}\left(\partial_{A_{0}} g_{\infty}\right), \\
& A_{\infty}^{0,1}=A_{0}^{0,1}+\left(\bar{\partial} g_{\infty}\right) g_{\infty}^{-1},
\end{aligned}
$$

and $A_{0}$ is itself real analytic. It follows that $g_{\infty}$ is real analytic, and therefore $h_{\infty}$ is also.

Now let $\mathcal{F}_{R}$ be the multiplier ideal sheaf associated to the sequence of metrics $\left\{H_{k}^{\prime}\right\}$ over $\tilde{X}=X \backslash\left\{x_{1}, \ldots, x_{p}\right\}$. Then, since $\hat{F}$ is bounded along the flow, and by the above, we can apply Theorem 2 to get a coherent sheaf $\mathcal{F} \subset \mathcal{E}$ over $\tilde{X}$. In fact, this defines a coherent subsheaf of $E$ over the whole of $X$. To see this, recall that a coherent subsheaf may be defined in the following way (see [UY], section 7 , for example). Let $U_{i}$ be a cover of $X$ so that $\left.E\right|_{U_{i}}$ is trivial and let $t_{i j}$ be the 
transition functions of $E$ defined on the intersections $U_{i} \cap U_{j}$. Then a coherent subsheaf of $E$ of rank $k$ can be defined to be a set $\left\{f_{i}\right\}$ of rational maps from $U_{i}$ to the Grassmannian $G(k, r)$ with transformation maps $t_{i j}$ on the overlaps. Thus, in particular, we can extend over a finite number of points in $X$.

We need the following lemma.

Lemma 4.2. There exists a positive constant $c$, independent of $k$, such that

$$
\left\|\operatorname{Tr} h_{k}\right\|_{L^{1}(X)} \geq c \sup _{X} \operatorname{Tr} h_{k}
$$

Proof. We use the inequality

$$
\triangle \log \operatorname{Tr} h_{k} \geq-\frac{1}{2}\left(\left|\hat{F}_{H_{0}}\right|+\left|\hat{F}_{H_{k}}\right|\right)
$$

from [S2], and the fact that $\hat{F}$ is uniformly bounded along the flow. We know that the Green's function $G=G(x, y)$ is bounded below by some constant $-A$. Then

$$
\begin{aligned}
\log \operatorname{Tr} h_{k}(x)= & \frac{1}{2 \pi} \int_{X} \log \operatorname{Tr} h_{k}(y) d V(y) \\
& +\int_{X}\left(-\triangle \log \operatorname{Tr} h_{k}\right)(y)(G(x, y)+A) d V(y) \\
\leq & \frac{1}{2 \pi} \int_{X} \log \operatorname{Tr} h_{k}(y) d V(y)+C \\
\leq & \log \left(\frac{1}{2 \pi} \int_{X} \operatorname{Tr} h_{k}(y) d V(y)\right)+C,
\end{aligned}
$$

where $C$ is independent of $k$. This proves the lemma.

Since $\operatorname{det} h_{k}=1$ along the flow, the above shows that at least one eigenvalue of $h_{k}$ must tend to infinity on a non-empty open set of $X$. Hence

$$
0<\operatorname{rank} \mathcal{F}<\operatorname{rank} \mathcal{E} .
$$

We will now show that $\mathcal{F}$ is destabilizing, which will give the contradiction. Note that $\mathcal{F}$ is a subbundle outside a subvariety $Z$ of codimension 1 . We will calculate the second fundamental form, following the method of [UY]. First observe that given a semi-positive self-adjoint (with respect to $H_{0}$ ) endomorphism $h$, one can define an endomorphism $h^{\sigma}$ by diagonalizing $h$ at a point in a unitary frame with respect to $H_{0}$ and then raising each matrix entry to the power $\sigma$. Then note that outside $Z \subset X$, the pointwise limit

$$
\pi=\lim _{\sigma \rightarrow 0}\left(I-h_{\infty}^{\sigma}\right)
$$

is the orthogonal projection of $E$ onto the subbundle defined by $\mathcal{F}$. Define the slopes $\mu(\mathcal{F})$ and $\mu(\mathcal{E})$ by

$$
\mu(\mathcal{E})=\frac{i}{2 \pi} \frac{\int_{X} \operatorname{Tr}\left(\hat{F}_{0}\right)}{\operatorname{rank}(\mathcal{E})} \quad \text { and } \quad \mu(\mathcal{F})=\frac{i}{2 \pi} \frac{\int_{X} \operatorname{Tr}\left(\hat{F}_{0}(\mathcal{F})\right)}{\operatorname{rank}(\mathcal{F})} .
$$

Note that $\mu(\mathcal{E})=i \lambda$. The following lemma completes the proof of Theorem 3 .

\section{Lemma 4.3.}

$$
\mu(\mathcal{F}) \geq \mu(\mathcal{E})
$$


Proof. First observe that for $0<\sigma \leq 1$,

$$
\begin{aligned}
\int_{X}\left|d h_{k}^{\prime \sigma}\right|_{H_{0}}^{2} & =2 \int_{X}\left|\partial_{0} h_{k}^{\prime \sigma}\right|_{H_{0}}^{2} \\
& \leq \int_{X}\left|\left(h_{k}^{\prime}\right)^{-\sigma / 2}\left(\partial_{0} h_{k}^{\prime \sigma}\right)\right|_{H_{0}}^{2} \\
& \leq \int_{X}\left\langle h_{k}^{-1}\left(\partial_{0} h_{k}\right), \partial_{0} h_{k}^{\prime \sigma}\right\rangle_{H_{0}} \\
& =\int_{X} i\left\langle\left(\hat{F}_{k}-\hat{F}_{0}\right), h_{k}^{\prime \sigma}\right\rangle_{H_{0}} \\
& \leq C
\end{aligned}
$$

where in the third line, we have used the pointwise inequality

$$
\left|h^{-\sigma / 2}\left(\partial_{0} h^{\sigma}\right)\right|_{H_{0}}^{2} \leq\left\langle h^{-1}\left(\partial_{0} h\right), \partial_{0} h^{\sigma}\right\rangle_{H_{0}}
$$

for $0<\sigma \leq 1$ and $h$ a positive self-adjoint endomorphism (see [UY], Lemma 4.1). Hence

$$
I-h_{k}^{\prime \sigma} \rightarrow \tilde{\pi}
$$

weakly in $L_{1}^{2}$, for some $\tilde{\pi}$ in $L_{1}^{2}$, taking the limit as $k$ tends to infinity and then as $\sigma$ tends to zero. Note that $\pi$ and $\tilde{\pi}$ must agree on $X \backslash Z$. Then

$$
\begin{aligned}
i \int_{X} \operatorname{Tr} \hat{F}_{0}(\mathcal{F}) & =i \int_{X} \operatorname{Tr}\left(\hat{F}_{0} \pi\right)-\int_{X}\left|\partial_{0} \pi\right|_{H_{0}}^{2} \\
& =i \int_{X} \operatorname{Tr}\left(\left(\hat{F}_{0}-\lambda I\right) \pi\right)+\int_{X} \operatorname{Tr}(\mu(\mathcal{E}) \pi)-\int_{X}\left|\partial_{0} \pi\right|_{H_{0}}^{2} \\
& =i \int_{X} \operatorname{Tr}\left(\left(\hat{F}_{0}-\lambda I\right) \pi\right)-\int_{X}\left|\partial_{0} \pi\right|_{H_{0}}^{2}+\operatorname{Vol}(X) \operatorname{rank}(\mathcal{F}) \mu(\mathcal{E}) .
\end{aligned}
$$

Hence $\mu(\mathcal{F}) \geq \mu(\mathcal{E})$ if and only if

$$
i \int_{X} \operatorname{Tr}\left(\left(\hat{F}_{0}-\lambda I\right) \pi\right) \geq \int_{X}\left|\partial_{0} \pi\right|_{H_{0}}^{2} .
$$

First, observe that using the inequality (4.1),

$$
\begin{aligned}
i \int_{X} \operatorname{Tr}\left(\left(\hat{F}_{0}-\lambda I\right) h_{k}^{\prime \sigma}\right) & =i \int_{X} \operatorname{Tr}\left(\left(\hat{F}_{k}-\lambda I\right) h_{k}^{\prime \sigma}\right)-\int_{X}\left\langle\bar{\partial}\left(h_{k}^{-1}\left(\partial_{0} h_{k}\right)\right), h_{k}^{\prime \sigma}\right\rangle_{H_{0}} \\
& \leq i \int_{X} \operatorname{Tr}\left(\left(\hat{F}_{k}-\lambda I\right) h_{k}^{\prime \sigma}\right)-\int_{X}\left|\partial_{0} h_{k}^{\prime \sigma}\right|_{H_{0}}^{2} .
\end{aligned}
$$

Then by lower semicontinuity,

$$
\begin{aligned}
\int_{X}\left|\partial_{0} \pi\right|_{H_{0}}^{2} & \leq \liminf _{\sigma \rightarrow 0} \liminf _{k \rightarrow \infty} \int_{X}\left|\partial_{0}\left(I-h_{k}^{\prime \sigma}\right)\right|_{H_{0}}^{2} \\
& =\liminf _{\sigma \rightarrow 0} \liminf _{k \rightarrow \infty} \int_{X}\left|\partial_{0} h_{k}^{\prime \sigma}\right|_{H_{0}}^{2} \\
& \leq \liminf _{\sigma \rightarrow 0} \liminf _{k \rightarrow \infty}\left(-i \int_{X} \operatorname{Tr}\left(\left(\hat{F}_{0}-\lambda I\right) h_{k}^{\prime \sigma}\right)+i \int_{X} \operatorname{Tr}\left(\left(\hat{F}_{k}-\lambda I\right) h_{k}^{\prime \sigma}\right)\right) \\
& =i \int_{X} \operatorname{Tr}\left(\left(\hat{F}_{0}-\lambda I\right) \pi\right)+\liminf _{\sigma \rightarrow 0} \liminf _{k \rightarrow \infty} i \int_{X} \operatorname{Tr}\left(\left(\hat{F}_{k}-\lambda I\right) h_{k}^{\prime \sigma}\right),
\end{aligned}
$$

using the fact that $\int_{X} \operatorname{Tr}\left(\hat{F}_{0}-\lambda I\right)=0$ and $I-h_{k}^{\prime \sigma} \rightarrow \tilde{\pi}$ weakly in $L_{1}^{2}$. Now since the Donaldson functional is bounded below, we know that $\int_{X}\left|\hat{F}_{k}-\lambda I\right|_{H_{k}}^{2}$ tends 
to zero as $k$ tends to infinity. This gives the desired inequality and completes the proof.

\section{ACKNOWLEDGEMENTS}

The author would like to thank his thesis advisor, D.H. Phong, for suggesting this problem, and for his constant advice and encouragement. The author is also indebted to Y-T. Siu, who visited Columbia University in the Fall of 2002 and gave a series of lectures [S5] on multiplier ideal sheaves. The ideas presented in those talks and in private discussions were invaluable in the shaping of this paper. In particular, Professor Siu proposed the use of a complex Frobenius theorem. The author is also grateful to Jacob Sturm for some very helpful suggestions, and to Zhiqin Lu and Nefton Pali for some useful discussions. The results of this paper are contained in the author's Ph.D. thesis We.

\section{REFERENCES}

[Bo] Bombieri, E. Algebraic values of meromorphic maps, Invent. Math., 10 (1970), 267-287 and Addendum, Invent. Math., 11 (1970), 163-166. MR0306201 (46:5328)

[DT] de Bartolomeis, P. and Tian, G. Stability of complex vector bundles, J. Diff. Geom. (2), 43 (1996), 231-275. MR1424426 (98f:32031)

[DC] de Cataldo, M.A.A. Singular hermitian metrics on vector bundles, J. reine angew. Math., 502 (1998), 93-122. MR 1647555 (2000c:32067)

[De] Demailly, J.-P. Effective bounds for very ample line bundles, Invent. Math., 124 (1996), 243-261. MR.1369417 (97a:32035)

[DeKo] Demailly, J.-P. and Kollár, J. Semi-continuity of complex singularity exponents and Kähler-Einstein metrics on Fano orbifolds, Ann. Sci. École Norm. Sup. (4), 34 (2001), 525-556. MR 1852009 (2002e:32032)

[D1] Donaldson, S.K. A new proof of a theorem of Narasimhan and Seshadri, J. Diff. Geom., 18 (1983), 269-277. MR.0710055 (85a:32036)

[D2] Donaldson, S.K. Anti self-dual Yang-Mills connections over complex algebraic surfaces and stable vector bundles, Proc. London Math. Soc. (3), 50 (1985), 1-26. MR0765366 (86h:58038)

[DoKr] Donaldson, S.K. and Kronheimer, P.B. The geometry of four manifolds, Clarendon Press, Oxford (1990). MR1079726 (92a:57036)

[HT] Hong, M.-C. and Tian, G. Asymptotical behaviour of the Yang-Mills flow and singular Yang-Mills connections, Math. Ann., 330 (2004), 441-472. MR2099188

[Ho] Hörmander, L. An introduction to complex analysis in several variables, North-Holland, Amsterdam, 3rd edition (1990). MR.1045639 (91a:32001)

[Ko] Kohn, J.J. Subellipticity of the $\bar{\partial}$-Neumann problem on pseudo-convex domains: sufficient conditions, Acta Math., 142 (1979), 79-122. MR0512213 (80d:32020)

[KM] Koszul, J.-L. and Malgrange, B. Sur certaines structures fibrés complexes, Arch. Math., 9 (1958), 102-109. MR0131882 (24:A1729)

[Ma] Malgrange, B. Ideals of differentiable functions, Oxford University Press (1966). MR0212575 (35:3446)

[Na] Nadel, A.M. Multiplier ideal sheaves and Kähler-Einstein metrics of positive scalar curvature, Ann. of Math., 132 (1990), 549-596. MR1078269 (92d:32038)

[NS] Narasimhan, M.S. and Seshadri, C.S. Stable and unitary vector bundles on compact Riemann surfaces, Ann. of Math., 82 (1965), 540-567. MR0184252 (32:1725)

[NN] Newlander, A. and Nirenberg, L. Complex analytic co-ordinates in almost complex manifolds, Ann. of Math., 65 (1957), 391-404. MR0088770 (19:577a)

[Pa] Pali, N. Faisceaux $\bar{\partial}$-cohérents sure les variétés complexes, preprint, arXiv: math.AG/0301146 (2003).

[Si] Simpson, C.T. Constructing variations of Hodge structure using Yang-Mills theory and applications to uniformization, J. Amer. Math. Soc. (4), 1 (1988), 867-918. MR0944577 (90e:58026) 
[S1] Siu, Y.-T. Analyticity of sets associated to Lelong numbers and the extension of closed positive currents, Invent. Math., 27 (1974), 53-156. MR0352516 (50:5003)

[S2] Siu, Y.-T. Lectures on Hermitian-Einstein metrics for stable bundles and Kähler-Einstein metrics, Birkhäuser Verlag, Basel (1987). MR0904673 (89d:32020)

[S3] Siu, Y.-T. An effective Matsusaka big theorem, Ann. Inst. Fourier, 43 (1993), 1387-1405. MR.1275204 (95f:32035)

[S4] Siu, Y.-T. Effective very ampleness, Invent. Math., 124 (1996), 563-571. MR1369428 (97a:32036)

[S5] Siu, Y.-T. Multiplier ideal sheaves in algebraic and complex geometry, Samuel Eilenberg Lectures at Columbia University (2002), unpublished.

[Sk] Skoda, H. Sous-ensembles analytiques d'ordre fini ou infini dans $\mathbf{C}^{n}$, Bull. Soc. Math. France, 100 (1972), 353-408. MR0352517 (50:5004)

[Uh] Uhlenbeck, K.K. Connections with $L^{p}$ bounds on curvature, Comm. Math. Phys., 83 (1982), 31-42. MR0648356 (83e:53035)

[UY] Uhlenbeck, K.K. and Yau, S.T. The existence of Hermitian Yang-Mills connections on stable bundles over Kähler manifolds, Comm. Pure \& Applied Math., 36 (1986), 257-293. MR:0861491 (88i:58154)

[We] Weinkove, B. The J-flow, the Mabuchi energy, the Yang-Mills flow and multiplier ideal sheaves, Ph.D. thesis, Columbia University, 2004.

Department of Mathematics, Harvard University, Cambridge, Massachusetts 02138 